\title{
Diminished oesophageal traction forces with swallowing in gastro-oesophageal reflux disease and in functional dysphagia
}

\author{
D Williams, D G Thompson, M Marples, L Heggie, T O’Hanrahan, J Bancewicz
}

\begin{abstract}
Relations between primary oesophageal peristaltic amplitude and traction force were studied in 30 normal volunteers, 12 patients with functional dysphagia, and 48 patients with gastro-oesophageal reflux disease, using a new intraluminal strain gauge device. Forces generated by swallowing in the normal oesophagus were $42(35-60) \mathrm{g}$ (median and interquartile range), a close positive correlation existing between traction force and contractile amplitude for each subject $(r=0.5(0.38-0.6)$. Traction force increased with increasing balloon volume from $62(50-73) \mathrm{g}$ at $2 \mathrm{ml}$ to 86 $(70-105) \mathrm{g}$ at $4 \mathrm{ml}(\mathrm{p}<0.05)$, indicating distension related modulation of peristaltic force. Patients with oesophagitis generated lower traction forces on swallowing $30(20-40) \mathrm{g}$ compared with the normal subjects $(\mathbf{p}<0.01)$, the degree of impairment being greatest in those patients with the most severe mucosal damage. Patients with gastro-oesophageal reflux without endoscopic oesophagitis also showed abnormal forces (32 22-38) g p $<0.01 v$ controls), which were similar to those patients with mild oesophagitis but were greater than those with severe oesophagitis $(p<0.05)$. In patients with functional dysphagia, forces were also impaired $(28(10-60) \mathrm{g} \mathrm{p}<0.05 v$ controls) despite normal standard manometry. Our results show that measurement of the traction force generated by primary peristalsis provides information about oesophageal neuromuscular function that is not demonstrable by manometry alone and can be abnormal in patients with oesophageal symptoms in whom standard techniques are normal. (Gut 1994; 35: 165-171)
\end{abstract}

In addition to the effect of gravity, transport of ingested material through the upright human oesophagus is fulfilled by swallow induced, aborally propagating, contractions, which are conventionally termed primary peristalsis.

Since the original descriptions by Meltzer,' intraluminal manometry has remained the preferred method for studying oesophageal peristaltic function in vivo. Substitution of perfused open tip catheters ${ }^{2}$ for balloons now provide a more accurate and convenient measure of intraluminal pressure, and the worldwide adoption of such systems has contributed valuable information to our understanding of human oesophageal function and dysfunction. ${ }^{3}$ It must be accepted, however, that peristaltic amplitude provides an incomplete description of oesophageal transport and while combined manometric and barium studies usually show a close relation between the amplitude of an intraluminal pressure wave and efficiency of clearance, with luminal clearance failing when amplitudes are low, ${ }^{45}$ the relation is less certain when amplitudes are more normal. Studies using isotopic methods also show this inexact relation, some patients with dysphagia exhibiting delayed isotope transit despite normal manometry. ${ }^{67}$ Conversely, normal isotope transit may be recorded in some patients with abnormal manometric wave forms. ${ }^{89}$

Direct measurement of the clearance force generated by primary peristalsis has been attempted both in animals ${ }^{10}$ and in humans. ${ }^{11}$ Earliest experiments measured the weight required to prevent an inflated balloon being propelled down the oesophagus by a swallow. ${ }^{10}$ Later studies used a 'mercury in silastic' strain gauge $^{12}$ to directly measure the force exerted on an intraluminal plastic sphere and showed that some patients with dysphagia could exhibit a reduced traction force in the presence of a normal contractile amplitude. Despite the importance of these studies, however, it seems that practical difficulties with the techniques have prevented their widespread introduction into clinical practice.

We now report a series of experiments measuring both manometric amplitude and oesophageal traction forces induced by swallowing using a simple miniature, intraluminal force transducer. The results of these studies, conducted both in normal subjects and in patients with oesophageal symptoms show that traction force responses to swallowing are now readily measurable in humans and that abnormalities can be found in association with symptoms, even when primary peristaltic amplitudes are normal.

\section{Subjects and methods}

\section{EQUIPMENT}

\section{The force detector}

The device consisted of a miniature strain gauge diaphragm (Gaeltec Ltd, Dunvegan, Isle of Skye) mounted on a catheter to which a silicone extension was attached by Kevlar threads (Fig 1). A $2 \mathrm{~cm}$ long latex balloon (HSC4 Precision Dippings Marketing, Stover Trading Estate, Yate, England) was attached to the extension and inflated to between 1 and $4 \mathrm{ml}$ by a channel running through the catheter. Because of their inelasticity, the Kevlar threads ensured that any swallowing induced traction forces acting on the balloon were transferred directly to the transducer. 


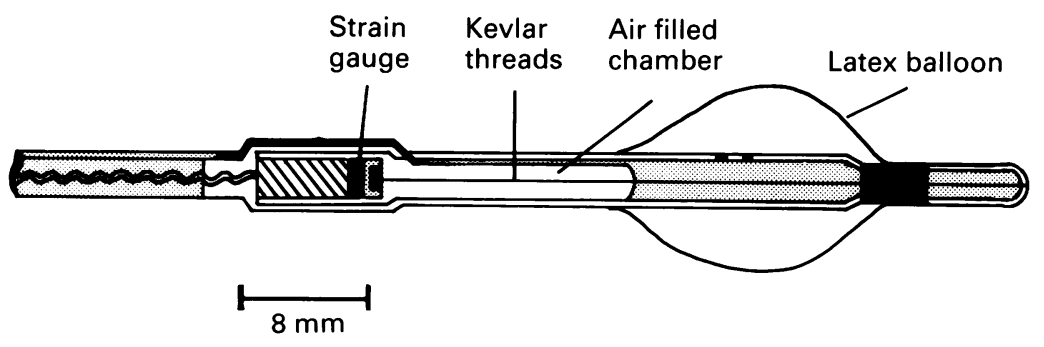

Figure 1: A simplified drawing of the traction catheter, showing the balloon bonded to the end of the catheter and attached directly to the strain gauge diaphragm by Kevlar threads.

Changes in the attached pre-amplifier voltage upon deformation of the diaphragm by known forces showed an output sensitivity of 45 microvolts/volt/g, with a linearity error of $7 \%$ between output and force over a range of $0-150 \mathrm{~g}$. The frequency of response to suspended weights was in excess of $150 \mathrm{~g} / \mathrm{s}$, with a hysteresis for applied weights of $1 \mathrm{~g}$ per $100 \mathrm{~g}$ reapplied weight and an output 'creep' of $2 \mathrm{~g}$ for an applied weight of $200 \mathrm{~g}$ for $20 \mathrm{~seconds}$. Forces over $500 \mathrm{~g}$ were not applied to avoid the possibility of irreversible damage to the transducer. The influence of bending the extension piece between the balloon and transducer was small (maximum error $5 \mathrm{~g}$ at 90 degrees).

Before and after each study the device was calibrated by suspending the catheter vertically, applying weights to the extension piece, and recording the voltage output changes on a chart recorder (Grass Instruments Co, Quincy, Mass, USA), calibrated so that $10 \mathrm{~mm}$ pen deflection was equivalent to $10 \mathrm{~g}$ force.

For combined measurement of contractile amplitudes and traction force, two $1 \mathrm{~mm}$ diameter perfusion tubes were attached to the catheter, the end of one being sited $1 \mathrm{~cm}$, the other $6 \mathrm{~cm}$, proximal to the balloon. Each was perfused with water at $0.6 \mathrm{ml} / \mathrm{min}$ using a standard pneumohydraulic system (Arndorfer Medical Specialties Inc, Wisconsin, USA). Complete occlusion of either channel gave a pressure response in excess of $300 \mathrm{~mm} \mathrm{Hg} / \mathrm{s}$, when measured by attached external force transducers (type: Statham P23-ID).

In the current experiments the balloon was inflated with between 1 and $4 \mathrm{ml}$ of air. These volumes were chosen on the basis of earlier studies $^{13}$ performed in our laboratory, which showed that they were below the threshold for induction of secondary (distension induced) peristalsis but were adequate to detect forces generated in the oesophagus by swallowing (primary peristalsis).

The device thus acted as a detector of swallowing induced peristaltic traction force for comparison with peristaltic amplitudes, without inducing any non-swallow related forces.

\section{Manometry}

For the conventional assessment of oesophageal contractile amplitude a four lumen catheter was constructed from $0.63 \mathrm{~mm}$ internal diameter polyethylene tubing (Portex Ltd, Hythe, Kent, England) the external catheter diameter being $3.5 \mathrm{~mm}$. Each lumen ended as a side hole oriented 90 degrees to the next and positioned 0 ,
3,8 , and $12 \mathrm{~cm}$ from the catheter tip. All four channels were perfused with water at a rate of 0.6 $\mathrm{ml} / \mathrm{min}$ using the pneumohydraulic perfusion system with a transducer (Statham P23-ID) attached to each to detect intraluminal pressure. The output of each transducer amplifier was recorded on the polygraph, run at a paper speed of $100 \mathrm{~mm} / \mathrm{min}$ and calibrated so that a pen deflection of $10 \mathrm{~mm}$ corresponded to a pressure change of $10 \mathrm{~mm} \mathrm{Hg}$.

\section{SUBJECTS}

\section{Normal volunteers}

The control studies were performed on 30 (18 female) asymptomatic volunteers, recruited from the hospital staff in accordance with Salford Health Authority ethics committee guidelines. The median age of the volunteers was 26 years (range 18-58). All volunteers first had standard manometric assessment of the oesophagus to confirm a normal oesophageal motor pattern before the traction studies were undertaken.

\section{Patient groups}

Gastro-oesophageal reflux: Forty eight patients (26 female, median age 48 (18-68) years) were studied. The diagnosis of gastro-oesophageal reflux disease was based on the finding of an intraoesophageal $\mathrm{pH}$ below 4 for more than $6 \%$ of a 24 hour study period. Thirty patients also had endoscopic evidence of oesophagitis. The endoscopic appearances were classified as follows: grade I (erythema and oedema) seven patients; grade II (isolated superficial mucosal lesions not affecting entire circumference) 11 patients; grade III (superficial mucosal damage affecting the entire circumference) six patients; grade IV (extensive mucosal damage and deeper ulceration of the wall) six patients. Patients with oesophageal strictures were not studied.

Functional dysphagia: Twelve patients (seven female, median age 46 (34-60) years) were studied. Entry criteria were persistent symptoms of food obstruction on swallowing for at least six months, together with a normal oesophagogastroscopy, normal standard oesophageal manometry, and normal 24 hour pH study. Six of the patients had, in addition, a barium swallow performed. In all six the examination was reported to be normal.

\section{STUDY PROTOCOLS}

All study protocols were presented to, and approved by the local ethics committee.

\section{Normal volunteer studies}

Study 1: Relation between contractile amplitude and traction force responses to swallowing - 12 volunteers were studied using both the traction catheter assembly and the manometry catheter, sited so that the most distal manometry channel was $1 \mathrm{~cm}$, and the midpoint of the balloon was $6 \mathrm{~cm}$, above the lower oesophageal sphincter. Both catheters were taped securely to the nose to prevent aboral movement. The balloon was first 
inflated with $1 \mathrm{ml}$ of air (balloon diameter 10 $\mathrm{mm}$ ) and 20 dry swallows were performed at 30 second intervals. This was followed by $2010 \mathrm{ml}$ water swallows again 30 seconds apart. If the subject swallowed inadvertently or coughed, the swallow was repeated after a further 30 second interval. To provide additional information about the relation between force and amplitude, a further 18 volunteers were studied. In these simplified studies, only the traction catheter was used, only $1 \mathrm{ml}$ of air placed in the balloon, only $1010 \mathrm{ml}$ water swallows were performed, and only the manometric events occurring $1 \mathrm{~cm}$ above the balloon were recorded. The results of these studies were then combined with data from the first 12 subjects and used as the normal data for comparison with the results of the patient studies.

Study 2: Effect of balloon volume on contractile amplitude and traction force - to assess the possible effect of balloon volume on relations between force and amplitude the protocol outlined above was repeated in eight volunteers using balloon volumes of $0,1,2,3$, and $4 \mathrm{ml}$ of air (giving maximum diameters $0,10,12,14$, and $16 \mathrm{~mm}$ ). These volumes were selected from the results of earlier studies on the effects of intraluminal distension on oesophageal motility, ${ }^{13}$ which showed them to be below the threshold required for stimulation of secondary (non-swallow induced) peristalsis. Each subject performed 10 dry and $1010 \mathrm{ml}$ water swallows at 30 second intervals, at each inflation volume.

Study 3: Measurement of amplitude and force at different sites in the oesophagus - 16 volunteers had further studies in which the balloon was positioned at $4,8,12$, and $16 \mathrm{~cm}$ above the lower oesophageal sphincter. On each occasion the balloon was inflated to $1 \mathrm{ml}$ and $1010 \mathrm{ml}$ water swallows were conducted at 30 second intervals as in study 1 .

\section{Patient studies}

Because the full protocol performed on the volunteers was lengthy and somewhat demanding, a simplified version was used on the patients. In all the patient studies therefore a modification of study 1 was conducted with measurement of traction force and contractile amplitude after $1010 \mathrm{ml}$ water swallows only. No studies of force and amplitude were conducted either during dry swallows or with change in balloon volume.

\section{DATA ANALYSIS}

All manometries were conducted by the same person (DW) to ensure consistency of data collection. The results were then checked independently by a further observer (MM or $\mathrm{LH}$ ) who was unaware of the origin of the tracing.

\section{Contractile amplitude}

The amplitude of a manometric wave was defined as the difference in value between the end expiratory baseline and peak pressure. Data are expressed in the text as millimetres of mercury ( $\mathrm{mm} \mathrm{Hg})$.

\section{Traction force}

This was defined as the difference between the end expiratory baseline value and the peak value of force exerted on the balloon during a swallow. The end expiratory value was chosen because it provided the most stable value. The transient rises in force that occurred with each inspiration were excluded from analysis (Fig 2). Data are expressed in grams $(\mathrm{g})$.

\section{STATISTICAL ANALYSIS}

To avoid assumptions regarding the distribution of the data, non-parametric statistics were used. ${ }^{14}$ Group comparisons were made using the Mann-Whitney U test and Kendall's rank correlation coefficient, the median value for each subject's dataset being selected for group comparisons. Kruskal-Wallis analysis was used to test the significance of multiple group differences.

The results are presented in the text as median and interquartile range, unless otherwise stated.

\section{Results}

\section{NORMAL VOLUNTEER STUDIES}

\section{General findings}

The general character of the traction waveform resembled that of the propagated pressure wave (Fig 2). At rest the oesophagus exhibited variations of pressure that increased with expiration and decreased with inspiration. The reverse pattern was seen on the traction record, with an increase of up to $10 \mathrm{~g}$ occurring upon inspiration, probably related to oesophageal lengthening with diaphragm descent. The respiratory variation was particularly evident in the distal oesophagus. Immediately before the arrival of a propagated pressure wave at the balloon there seemed to be a transient fall in traction force.

\section{Effect of balloon volume on amplitude and force}

Increasing the balloon volume was associated with a progressive increase in force exerted on the transducer by both wet and dry swallows from $10(4-16) \mathrm{g}$ and $6(4-12) \mathrm{g}$ respectively
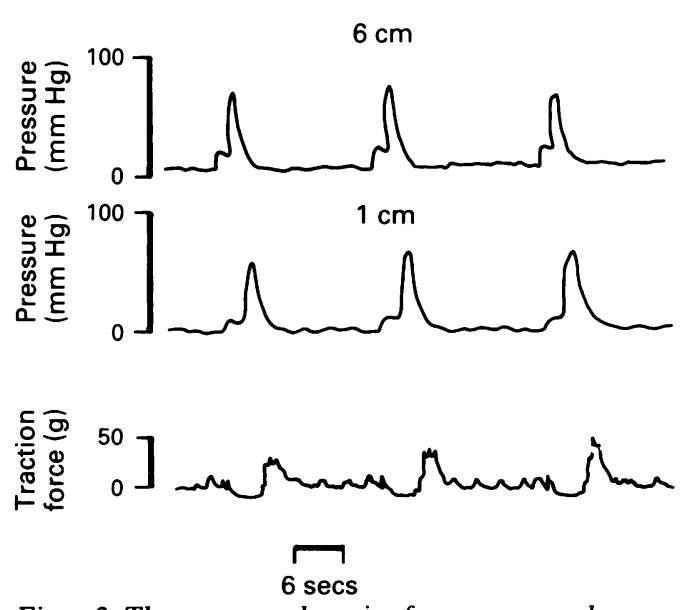

Figure 2: The pressure and traction force patterns to three water swallows in a normal subject. The pressure recording sites are 1 and $6 \mathrm{~cm}$ proximal to the balloon. 


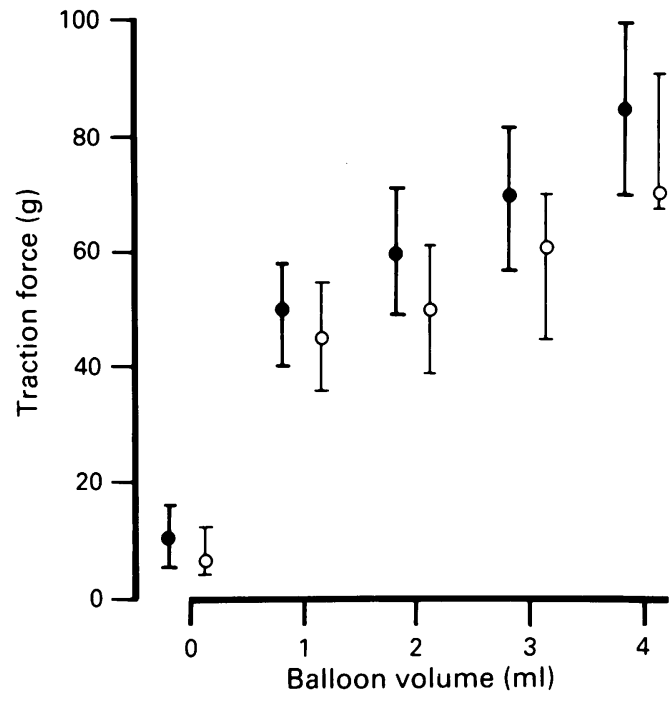

Figure 3: The effect of balloon volume on the traction force in eight volunteers. Symbols represent median and interquartile range for dry swallows (open circles) and wet swallows (solid circles).

when the balloon was deflated, to $50(40-60) \mathrm{g}$ and $45(36-55) \mathrm{g}$ at $1 \mathrm{ml}$ and $85(75-100) \mathrm{g}$ and 70 (68-91) g at $4 \mathrm{ml}$ ( $\mathrm{p}<0.01 \mathrm{Kruskal}-\mathrm{Wallis})$ (Fig 3). A similar volume related increase in contractile amplitude to dry swallows was also seen at the $1 \mathrm{~cm}$ site but not at the $6 \mathrm{~cm}$ level (Fig 4) $\mathrm{p}<0.01$. No change in contractile amplitude with balloon volume was apparent for water swallows, values being $54(47-65) \mathrm{mm} \mathrm{Hg}$ at $1 \mathrm{ml}$ and $58(50-72) \mathrm{mm} \mathrm{Hg}$ at $4 \mathrm{ml}(\mathrm{p}>0 \cdot 05)$.

\section{Variation in amplitude and force at different sites in} the oesophagus

When amplitude and force were compared at different sites, parallel site dependent changes

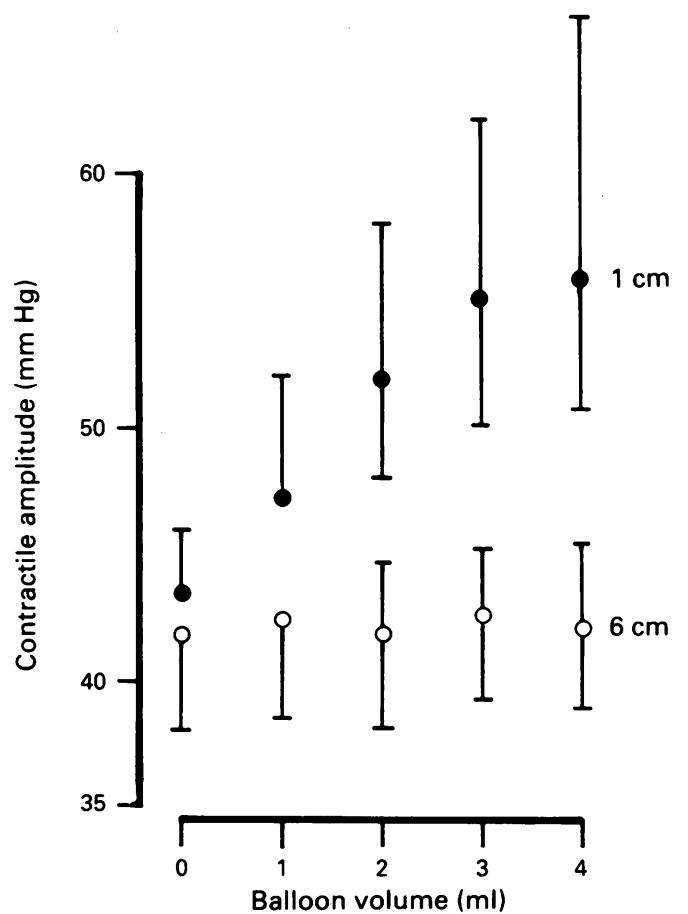

Figure 4: The effect of increasing balloon volume on contractile amplitudes to dry swallows at sites 1 and $6 \mathrm{~cm}$ above the balloon in eight volunteers. Note the increase in amplitude with increasing volume at the $1 \mathrm{~cm}$ level $(p<0.01)$ but not at the $6 \mathrm{~cm}$ level. Symbols show median and interquartile range.

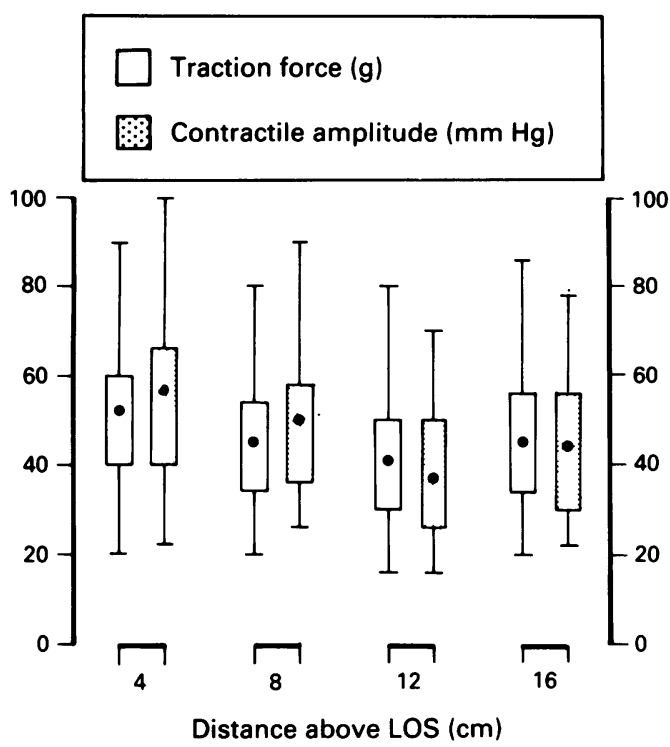

Figure 5: The values for force and amplitude to wet swallows measured at different sites in the oesophagus in 16 normal subjects. Symbols represent the median, interquartile range, and range. $L O S=$ lower, oesophageal sphincter.

were seen (Fig 5). In the most distal oesophagus ( $4 \mathrm{~cm}$ above the lower oesophageal sphincter) amplitudes of $55(44-70) \mathrm{mm} \mathrm{Hg}$ and forces of 52 $(40-65) \mathrm{g}$ were found. In the more proximal oesophagus (12 $\mathrm{cm}$ above the sphincter) values were lower at $48(40-58) \mathrm{mm} \mathrm{Hg}$ and $44(36-54)$ $\mathrm{g}(\mathrm{p}<0.01 v 4 \mathrm{~cm})$.

\section{Amplitude and force relations}

Dry swallows induced a force of $42(35-60) \mathrm{g}$ with amplitudes of $47(40-53) \mathrm{mm} \mathrm{Hg}$ at $1 \mathrm{~cm}$, and $42(38-52) \mathrm{mm} \mathrm{Hg}$ at $6 \mathrm{~cm}$ proximal to the balloon. Water swallows generated greater forces $(50(41-70) \mathrm{g})$ and amplitudes $(58(52-80) \mathrm{mm}$ $\mathrm{Hg}$ at $1 \mathrm{~cm}$ and $52(45-64) \mathrm{mm} \mathrm{Hg}$ at $6 \mathrm{~cm})$ than dry swallows. A positive correlation existed between force and amplitude $1 \mathrm{~cm}$ proximal to the balloon for each subject studied (Fig 6, Table I). No consistent correlations between force and amplitude were found $6 \mathrm{~cm}$ proximal to the balloon.

Values for the combined group of 30 volun-

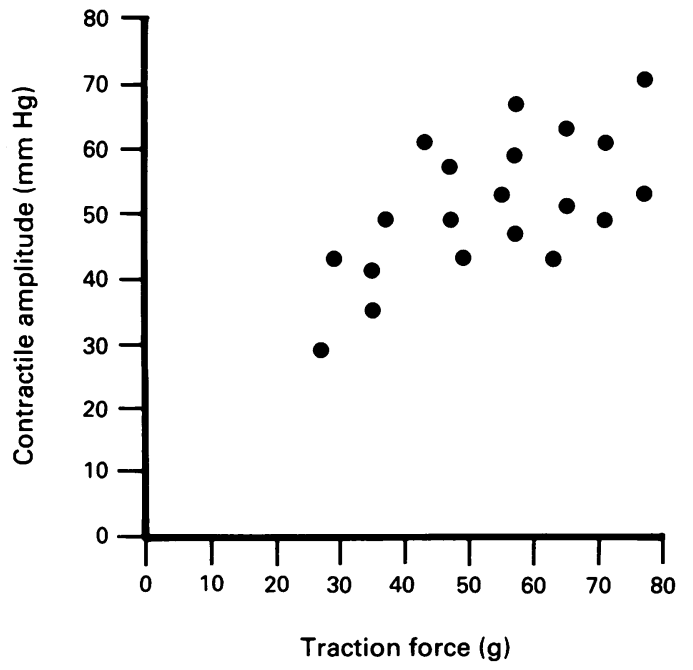

Figure 6: The correlation between force and amplitude $(1 \mathrm{~cm}$ proximal to the balloon) obtained from 20 water swallows in one normal subject. Correlation coefficient $=0 \cdot 7$. 
TABLE I Correlation coefficients for relation between contractile amplitude and traction force for the normal subjects

\begin{tabular}{lll}
\hline \multirow{2}{*}{$\begin{array}{l}\text { Distance above site } \\
\text { of distension }\end{array}$} & Type of swallow & \\
\cline { 2 - 3 } & Dry $(n=12)$ & Water $(n=30)$ \\
\hline $1 \mathrm{~cm}$ & $0.5(0.38-0.6)(0 \cdot 3-0.8), \mathrm{p}=0.05$ & $0.53(0.42-0.63)(0 \cdot 36-0.8), \mathrm{p}=0.05$ \\
$6 \mathrm{~cm}$ & $0 \cdot 1(0.03-0.2)(0.02-0.4), \mathrm{p}=0.3$ & $0.09(0.02-0.2)(0.02-0 \cdot 3), \mathrm{p}=0.4$ \\
\hline
\end{tabular}

Data represent median (interquartile values) and (range), respectively.

teers were similar, with amplitudes of $60(50-64)$ $\mathrm{mm} \mathrm{Hg}$ and forces of $42(35-60) \mathrm{g}$ to water swallows.

\section{GASTRO-OESOPHAGEAL REFLUX PATIENTS}

In patients with reflux but without oesophagitis, both contractile amplitudes to dry swallows and traction forces were consistently reduced at 50 (45-60) $\mathrm{mm} \mathrm{Hg}$ ( $\mathrm{p}=0.05 v$ normal controls and $32(22-38) \mathrm{g}(\mathrm{p}<0.01 v$ normal controls) respectively. As in the volunteer studies there was no consistent relation between amplitude and force after 10 water swallows $(r=0 \cdot 1, p=0 \cdot 6)$.

Patients with oesophagitis also had lower contractile amplitudes, $45(25-60) \mathrm{mm} \mathrm{Hg}$ and traction forces, $30(20-40) \mathrm{g}(\mathrm{p}<0.01$ compared with control values) but their results were similar to those for patients with reflux without oesophagitis ( $p>0.5$ for both comparisons).

Six of 30 patients were found to have normal peristaltic amplitudes yet impaired forces (Fig 7).

When the oesophagitis patients were divided into two groups according to severity of oesophagitis, consistent differences were found in amplitude and force between those patients with the mildest oesophagitis (grades I and II) and those with the most severe oesophagitis (grades III and IV), $\mathrm{p}<0.01$ (Table II). Values for the patients with reflux but no oesophagitis were similar to the group containing grade I and II but were greater than the group containing grades III and IV $(\mathrm{p}<0.05)$.

between amplitude and force in 30 patients with

oesophagitis. Each point represents the median value of 10 measurements for $a$ patient. The range for the normal subjects is shown by the hatched area. Note the presence of six patients with normal amplitudes but impaired force.

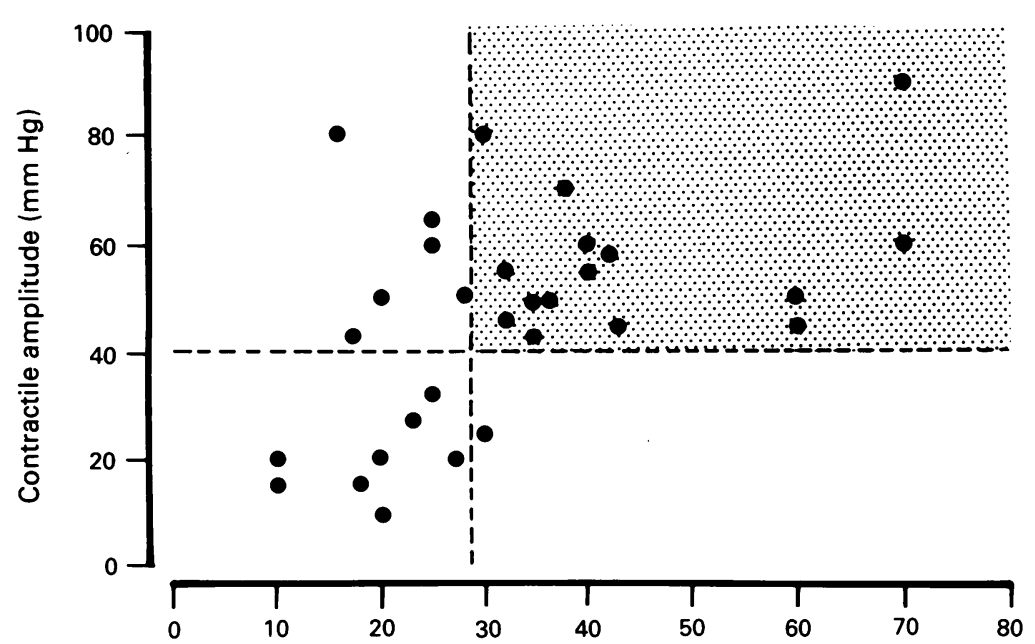

Traction force $(g)$ contractile amplitudes $(56$ (42-80) $\mathrm{mm} \mathrm{Hg}$ ( $\mathrm{p}>0.5 v$ normal) (Fig 8). In six of 12 patients, values for traction force were below the lower limit of the normal range.

\section{RELATIONS BETWEEN AGE AND PERISTALTIC} FUNCTION

Because the ages of the normal volunteers differed from the patient groups it was questioned whether age might contribute to the impaired force generation. Correlations between age, peristaltic force, and amplitude were therefore sought, but none were found for any group studied.

\section{Discussion}

It is apparent from our results that the change in intraluminal pressure detectable by manometry is not the only factor determining the traction force generated by a propagated oesophageal contraction. Other important factors not detected by manometry include the magnitude of the pressure differential across the bolus, and distal resistance forces ${ }^{15}$ analagous to the preload and afterload responses of cardiac muscle. In addition, longitudinal muscle contraction probably contributes to force generation as bolus transport seems to be enhanced by oesophageal shortening. ${ }^{16}$ Because it does not produce lumen occlusion, however, this longitudinal muscle contribution to clearance is unlikely to be detected.

The results of the studies using varying balloon volumes show that both the traction force and the amplitude of swallow induced pressure waves are modulated by degrees of luminal distension that are insufficient to induce secondary peristalsis. This suggests that local modulation of swallow induced peristalsis occurs in response to mechanoreceptor stimulation probably affecting enteric neural pathways, a conjecture that is consistent with our own finding and previous reports showing that water swallows, which promote some bolus distension of the oesophageal wall, generate greater contractile amplitudes than dry swallows, which do not. ${ }^{12} 17$

The absence of any distension associated change in contractile amplitude with water swallows was unexpected. The most probable explanation is that the volume of water used (10 $\mathrm{ml}$ ) was sufficient to induce a distension related enhancement of peristalsis, which was equal to that produced by the balloon. In support of this suggestion is the finding that water swallow related contractile amplitudes at both $1 \mathrm{~m}$ and $6 \mathrm{~cm}$ proximal to the balloon were similar in magnitude to those dry swallows enhanced by $4 \mathrm{ml}$ balloon distension. This suggestion could be further explored by studies of peristaltic amplitude during water swallows of varying volume to see whether the relation was also evident at smaller volumes.

Although peristaltic amplitude is not the only factor determining propulsive force, our results do show, as might be expected, that for each normal subject a positive correlation between the two does exist. Between normal subjects, how- 
TABLE II Contractile amplitudes and traction forces to water swallows in the lower oesophagus in patients with oesophagitis

\begin{tabular}{lllll}
\hline $\begin{array}{l}\text { Grade No of } \\
\text { patients }\end{array}$ & $I$ & $I I$ & $I I I$ & $I V$ \\
\hline $\begin{array}{c}\text { Amplitude } \\
(\text { mm Hg) }\end{array}$ & $57(50-72)(40-90)$ & $55(45-68)(28-80)$ & $48(30-53)(20-60)$ & $20(14-28)(10-40)$ \\
Force $(\mathrm{g})$ & $35(30-55)(25-70)$ & $30(25-50)(16-70)$ & $31(22-36)(10-40)$ & $19(15-26)(10-35)$
\end{tabular}

This table shows the differences between contractile amplitude and traction force in the lower oesophagus with severity of oesophagitis. Number show median, interquartile values, and range respectively.

ever, there seems to be a poor relation between force and amplitude suggesting that other, undetermined, factors are probably operating.

The transient fall in traction force noted immediately before the arrival of the propagated wave is of interest because it suggests that a wave of inhibition of muscle tone precedes each peristalsis. This preceding wave of inhibition is not detected by standard manometry but has been recently reported using a technique designed specifically to measure resting oesophageal muscle tone. ${ }^{18}$

The results of the studies on gastro-oesophageal reflux patients show consistent abnormalities of traction force. Those patients with reflux without oesophagitis showed diminished traction forces in the distal oesophagus despite normal contractile amplitudes while those with endoscopic oesophagitis had lower contractile amplitudes in addition to the traction force abnormality. Although the number of patients in our study was too small to permit a relation between traction force and severity of oesophagitis to be explored in detail, it was evident that patients with the most severe oesophagitis did show the lowest traction forces of the group suggesting either, that impaired acid clearance may in some way contribute to the development of mucosal damage or, that oesophageal neuromuscular damage by the inflammatory process, impairs force generation. It is of further relevance to note that some of the least severe oesophagitis patients also showed normal contractile amplitudes but abnormally low traction forces, suggesting in addition, that the presence

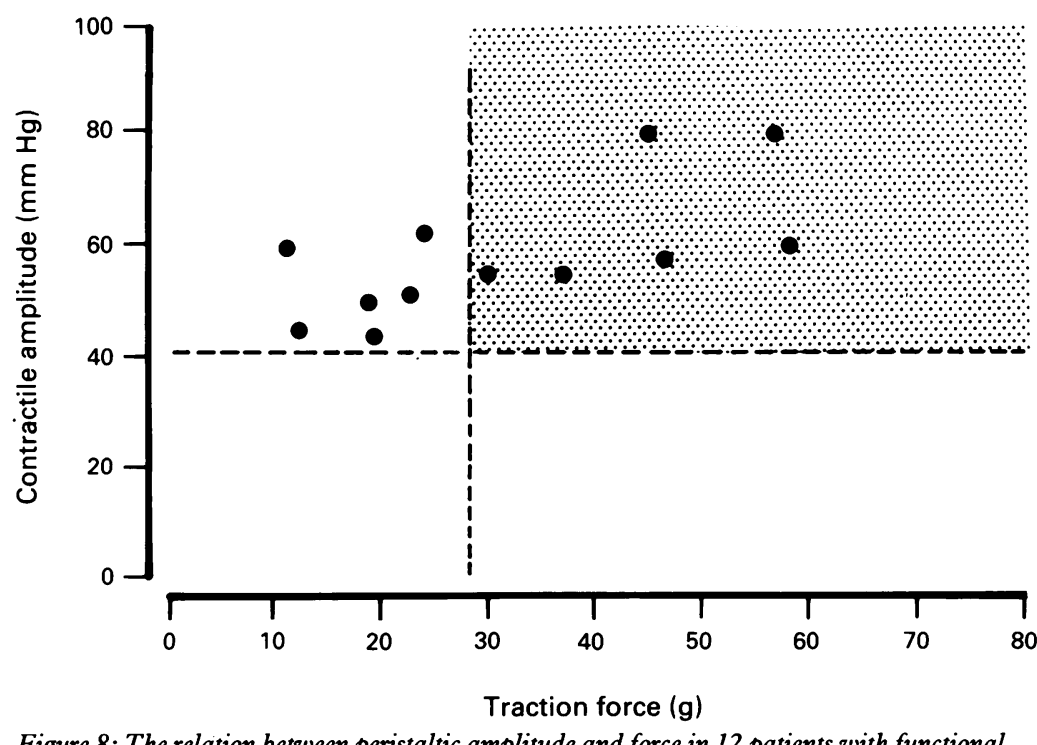

Figure 8: The relation between peristaltic amplitude and force in 12 patients with functional dysphagia. The control range is shown by the hatched area. Each point represents the median value of 10 measurements for a patient. Note the presence of six patients with normal amplitudes but impaired force. of oesophagitis is more directly related to impaired swallowing induced clearance force than contraction amplitude.

In patients with functional dysphagia the diagnostic advantage of traction force assessment is also shown by the finding that in half of the patients traction forces generated were abnormally low despite normal contractile amplitudes. These findings are compatible with earlier studies showing weak propulsive forces ${ }^{12}$ and delayed isotope transit ${ }^{7}$ in patients with functional dysphagia.

The most probable explanation for the dissociation of manometric amplitude and traction force in both patient groups lies in the inability of routine manometric assessment to identify those more subtle forms of oesophageal motor dysfunction, such as abnormalities of longitudinal muscle contraction, which are known to play an important part in luminal clearance. When these more subtle abnormalities are specifically sought, for example, by performing manometry during meal ingestion, abnormalities of both contractile function and clearance can be detected. ${ }^{919} 20$

Because our studies compared two different techniques in disorders for which an accepted diagnostic gold standard is lacking, it could be argued that the discrepant results could be equally explained by a false positive error in the traction data and not a false negative manometric result. This suggestion seems unlikely, however, because the symptoms suffered by the patients undoubtedly shows that they had difficulties with oesophageal clearance. The pathophysiology of symptoms in those patients with both normal contractile amplitudes and traction forces remains unexplained, however, and requires further comparison of traction forces with other independent measures of clearance, such as isotope transit, to determine how closely low traction forces are related to delayed luminal clearance.

In conclusion, our findings show that measurement of the traction force generated by swallowing, complements information gained by standard manometry and can identify abnormalities in patients with oesophageal symptoms in whom no manometric abnormality is detectable. These findings, together with similar more recent data from other workers using similar techniques $^{21} 22$ justify its further exploration in the assessment of oesophageal pathophysiology.

The authors wish to thank Mrs J Young for preparing the manuscript and Gaeltec Ltd for help in the design and construcmanuscript and Gaeltec Ltd for help in the design and construcwas conducted with the help of a project grant from the NW Regional Health Authority, England. This work was presented in Regional Health Authority, England. This work was presented in
part at the 1990 meeting of the American Gastroenterological

1 Meltzer SJ. On the causes of the orderly progress of the peristaltic movements in the oesophagus. Am F Physiol 1899; 2: 266-72.

2 Pert JH, Davidson M, Almy TP, Sleisinger MV. Esophageal catheterisation studies. 1 . The mechanism of swallowing in normal subjects with particular reference to the vestibule normal subjects with particular reference to the vestibule
(esophago-gastric sphincter). $\mathcal{F}$ Clin Invest 1959; 38: 397406.

3 Richter JE. Normal values of esophageal manometry. In: Castell DO, Richter JE, Dalton CB, eds. Esophageal motility testing. New York: Elsevier, 1987: 79-90.

4 Kahrilas PJ, Dodds WJ, Hogan WJ. Effect of peristaltic dysfunction on esophageal volume clearance. Gastroenterology 1988; 84: 73-80. 
5 Hewson EG, Ott DJ, Dalton CB, Chen YM, Wu WC, Richter JE. Manometry and radiology. Complementary studies in the assessment of esophageal motility disorders. Gastroenterology 1990; 98: 626-32.

6 Russell COH, Hill LD, Holmes ER, Hull DA, Cannon R, Pope CE. Radionuclide transit. A sensitive screening test for oesophageal dysfunction. Gastroenterology 1981; 80: 887-92.

Russell COH, Whelan G. Oesophageal manometry: how well does it predict oesophageal function? Gut 1987; 28: 940-5.

8 Mughal MM, Marples M, Bancewicz J. Scintigraphic assessment of oesophageal motility: what does it show and how ment of oesophageal motility: what

9 Kjellan G, Svedberg JB, Tibbling L. Solid bolus transit by esophageal scintigraphy in patients with dysphagia and normal manometry and radiology. Dig Dis Sci 1984; 29: 1-5.

10 Hwang K. Mechanism of transportation of the content of the esophagus. F Appl Physiol 1954; 6: 781-6.

11 Ingelfinger FJ. Esophageal motility. Physiol Rev 1958; 38: 533-84.

12 Pope CE, Horton PF. Intraluminal force transducer measurements of human oesophageal peristalsis. Gut 1972; 13: 464 70.

13 Williams D, Thompson DG, Marples $M$, Heggie L, O'Hanrahan T, Mani V, et al. Identification of an abnormal esophageal clearance response to intraluminal distension in
patients with esophagitis. Gastroenterology 1992; 103: 943patient
14 Leach C. Introduction to statistics. A non parametric approach for the social sciences. Chichester: J Wiley, 1979: 148-68.

15 Brasseur JG. A fluid mechanical perspective on esophageal bolus transport. Dysphagia 1987; 2: 32-9.

16 Ren J, Schultze-Delrieu K. Movement of wax particles by contractions in the isolated opossum esophagus. $A m \mathcal{F}$ Physiol 1990; 258: G164-70.

17 Schoen HJ, Morris DW, Cohen S. Esophageal peristaltic force in man. Response to mechanical and pharmacological in man. Response to mechanical and

18 Sifrim D, Janssens J, Vantrappen G, Coulie B. A wave of inhibition precedes primary esophageal contractions in man and is absent or abnormal in some patients with primary esophageal motility disorders. Gastroenterology 1991; 100: Al61.

19 Mellow MH. Esophageal motility during food ingestion: a physiologic test of esophageal motor function. Gastroenterology 1982; 85: 570-7.

20 Howard PJ, Pryde A, Heading RC. Oesophageal manometry during eating in the investigation of patients with chest pain or dysphagia Gut 1989; 30: 1179-86.

21 Mangan MJ, Kahrilas PJ, Flanagan E. Esophageal propulsive force: relationship to peristaltic function and bolus diameter. Gastroenterology 1991; 100: A467.

22 Russell COH, Bright N, Buthpitiya G, Alexander L, Walton $\mathrm{C}$, Whelan $\mathrm{G}$. Oesophageal propulsive force and its relation to manometric pressure. Gut 1992; 33: 727-32. 